\title{
Parametric decay of oblique arc-polarized Alfvén waves
}

\author{
Luca Del Zanna \\ Dipartimento di Astronomia e Scienza dello Spazio, Università di Firenze, Italy.
}

\begin{abstract}
The long-term nonlinear evolution of monochromatic large-amplitude Alfvén waves in oblique propagation is studied via one-dimensional magnetohydrodynamic (MHD) numerical simulations. Arc-polarized waves, which are exact solutions for non-parallel propagation and are observed in solar wind data, are shown to be subject to parametric decay instabilities just like the well studied case of circularly polarized waves, not only when arc polarization is used as initial condition but also when this state is reached after nonlinear evolution of linearly polarized waves.
\end{abstract}

\section{Introduction}

The low-frequency part of fluctuations spectra in highspeed and polar regions of the solar wind is known to be dominated by large-amplitude Alfvén waves propagating outwards. Magnetic field oscillations are never circularly polarized, as would be natural for parallel propagating Alfvén waves preserving $B \sim$ const, but rather show a spherical arc-type polarization, with the tip of $\delta \mathbf{B}$ describing circular arcs (rotations are usually less than $180^{\circ}$ ) on the surface of an imaginary sphere with radius $B=|\mathbf{B}|[$ Riley et al., 1995; 1996; Tsurutani and Ho, 1999, and references therein]. According to Riley et al. [1996], who performed a systematic analysis by studying Ulysses fast-stream data in the ecliptic plane, up to $10 \%$ of Alfvénic modes are pure arcpolarized waves, meaning that the plane containing the maximum and intermediate variance directions remains fixed in time. The majority of the events was best explained by planar (1-D) arc-polarized Alfvén waves propagating obliquely to the background magnetic field and by embedded phasesteepened structures (rotational discontinuities: RDs).

Arc polarization for large-amplitude oblique Alfvénic modes was first proposed by Barnes and Hollweg [1974, $(\mathrm{BH})$ ] who showed, by performing a second-order expansion of the 1-D MHD nonlinear equations, that a monochromatic Alfvén wave which is initially linearly polarized develops another oscillatory component achieving arc polarization and $B^{2}=$ const. Magnetoacoustic modes should also form in the process, but Landau damping was proposed to be efficient in the almost collisionless solar wind plasma so as to leave the exact Alfvénic $B^{2}=$ const mode dominant, as observed. This was confirmed by Vasquez and Hollweg [1996 (VH)] by using hybrid simulations, who indeed found asymptotic Alfvénic states of approximate arc polarization and constant $B$ (both waves and RDs for sufficiently high amplitudes). Another important result found by $\mathrm{VH}$ is the dependence on the plasma beta: for $\beta>1$ the density fluctuations created initially are rapidly damped, while for

Copyright 2001 by the American Geophysical Union.

Paper number 2001GL012911. 0094-8276/01/2001GL012911\$05.00 $\beta<1$ they persist over many Alfvénic periods and are even seen to increase, due to parametric effects according to the authors. To check this point, they also performed one simulation with initial constant magnetic field (arc polarization) and indeed that mode was seen to grow exponentially in time.

Parametric decay of Alfvén waves is a very important process occurring in low-beta plasmas, where the instability growth rate is the highest. An Alfvén wave decays into a compressive mode (slow magnetoacoustic or sound wave in parallel propagation) and into a backscattered Alfvénic mode, with a lower wave number than the mother wave. This decay has been extensively studied in the literature (see [Del Zanna et al., 2001 (DZ)] for references) and has been proposed as a possible mechanism to produce the inward propagating Alfvénic component measured in the solar wind (e.g. [Inhester, 1990] for estimates and comparisons with other possible mechanisms). In DZ the nonlinear saturation phase is shown to occur always when the produced magnetoacoustic modes steepen into shocks, causing about half of the total Alfvénic energy to go in the daughter modes and into heat. When density fluctuations reach the highest level (just before saturation) the cross helicity suffers a rather rapid decrease: in high-beta conditions an asymptotic balance between outward and inward modes is found, as observed with increasing heliocentric distance in the solar wind, while for low-beta conditions the cross helicity usually reverses its sign (the backscattered wave becomes the dominant mode) and sometimes even multiple decays are observed. Similar results are also found in 2-D and 3-D and even in non-periodic domains, confirming the robustness and universality of the parametric decay process.

In the present paper the constraints of parallel propagation and circular polarization, that are usually assumed for simplicity but that do not seem apply to solar wind conditions, will be relaxed. Stability studies of such Alfvénic structures may also prove useful to test the derivative and kinetic nonlinear Schrödinger equation approximations (DNLS and KNLS, see [Medvedev et al., 2000] and references therein), that allow these solutions and predict some aspects of their nonlinear evolution (like formation of arc-polarized waves), but where compressive wave-wave interactions like parametric decay instability are not included.

\section{Simulation results}

As in DZ, the MHD equations neglecting dispersive effects will be solved numerically via a new high-order upwind code [Londrillo and Del Zanna, 2000]. The numerical box (256 grid points will be used in all runs) is a periodic 1-D domain of length $L=2 \pi$. This will be labeled with the $z$ coordinate and we will take $\mathbf{k}_{0}=k_{0} \mathbf{e}_{z}$ and $\mathbf{B}_{0}=B_{0} \sin \theta \mathbf{e}_{y}+B_{0} \cos \theta \mathbf{e}_{z}$. The initial magnetic and velocity fluctuations along the direction of maximum 
variance are:

$$
B_{x}=\delta B_{x}=\eta B_{0} \cos \left(k_{0} z\right), \quad v_{x}=-\delta B_{x} / \sqrt{\rho_{0}},
$$

corresponding to an Alfvénic mode propagating in the positive $z$ direction with phase speed $\omega_{0} / k_{0}=v_{A z}=\cos \theta$, where both the background field $B_{0}$ and density $\rho_{0}$ are normalized to unity, the kinetic pressure is $p_{0}=(3 / 5) \beta$ $\left(\beta=c_{s}^{2} / v_{A}^{2}\right)$, and $k_{0}$ is simply the number of wavelengths in the simulation box, while the wave period will be $P_{0}=$ $2 \pi /\left(k_{0} \cos \theta\right)$ in these units.

\subsection{Arc-polarized waves}

In this section an exact arc-polarized wave is set up as initial condition. To achieve an overall constant- $B$ state, an additional fluctuation has to be added to the background field in the $y$ direction, that is:

$$
B_{y}=B_{0 y}+\delta B_{y}=\sqrt{C^{2}-B_{x}^{2}}, \quad v_{y}=-\delta B_{y} / \sqrt{\rho_{0}},
$$

where $C^{2}=B_{x}^{2}+B_{y}^{2}=B^{2}-B_{0}^{2}$ is the constant squared module of the transverse magnetic field and is obtained by imposing $<\delta B_{y}>=0$, that is by solving the nonlinear equation

$$
\frac{1}{\xi} \int_{0}^{\pi / 2} \sqrt{1-\xi^{2} \cos ^{2} \varphi} \mathrm{d} \varphi=\frac{\pi}{2} \frac{\sin \theta}{\eta}
$$

where $\xi=\eta B_{0} / C$ is the argument of the complete elliptic integral appearing on the left-hand side (this result holds regardless of $k_{0}$ but only for values of the right-hand side greater than unity, thus giving a minimum critical angle defined by $\sin \theta_{c}=2 \eta / \pi$, see $\mathrm{BH}$ and $\mathrm{VH}$ ). Under the present conditions, the magnetic field fluctuations describe a perfect arc in the $x-y$ plane of amplitude less than $180^{\circ}$ (thus less than half of the circle corresponding to circular polarization). In order to start the instability, a $10^{-4} \mathrm{rms}$ density fluctuation is imposed at $t=0$. In the solar wind small compressive fluctuations are always present, possibly arising due to the interaction with transverse quasi-2D small-scale MHD turbulence (e.g. [Leamon et al., 2000] and references therein). To compare with the results in DZ, the same choice of $\eta$ and $\beta$ will be assumed, with a set of different values for the angle $\theta$.

By looking at the time histories of the rms quantities, it is easy to see that the parametric instability behaves exactly in the same way as in the case of circular polarization. In Table 1 the parameters used and the measured growth rates $\gamma$ for $\theta=60^{\circ}$ are shown. The analytical values corresponding to circular polarization are also reported $\left(\theta=0^{\circ}\right)$. The initial wave number is always $k_{0}=4$, whereas $k_{c}$ and $k_{t}^{-}$are the wave numbers of the daughter compressive mode and backscattered transverse Alfvénic mode, respectively. As in the circular polarization case, runs A, B and C yield

Table 1. Simulation parameters for the three runs, labeled A, $\mathrm{B}$ and $\mathrm{C}$ (see text). The growth rates are to be compared with mother wave periods $P_{0}=\pi / 2$ for $\theta=0^{\circ}$ and $P_{0}=\pi$ for $\theta=60^{\circ}$.

\begin{tabular}{cccccccc}
\hline run & $\eta$ & $\beta$ & $k_{0}$ & $k_{c}$ & $k_{t}^{-}$ & $\gamma\left(0^{\circ}\right)$ & $\gamma\left(60^{\circ}\right)$ \\
\hline $\mathrm{A}$ & 0.2 & 0.1 & 4 & 6 & 2 & 0.41 & 0.135 \\
$\mathrm{~B}$ & 0.5 & 0.5 & 4 & 5 & 1 & 0.39 & 0.113 \\
$\mathrm{C}$ & 1.0 & 1.2 & 4 & 5 & 1 & 0.41 & 0.119 \\
\hline
\end{tabular}
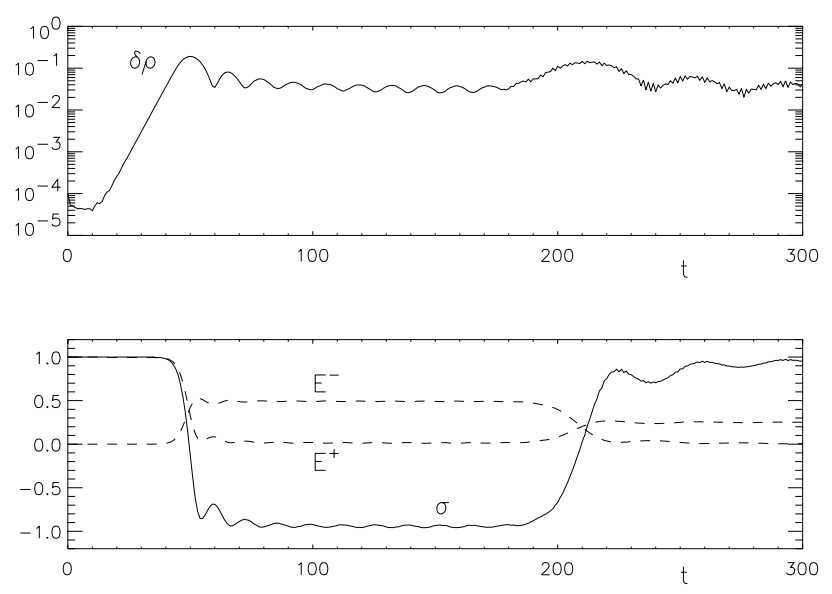

Figure 1. Time histories of rms density fluctuations (top), normalized cross helicity $\sigma$ and normalized Elsässer energies $E^{ \pm}$. Parametric decay occurs twice, at $t=50$ and $t=210$. Here run A parameters with $\theta=30^{\circ}$ are employed.

very similar growth rates for all angles, so that the increase with increasing amplitude and the decrease with increasing plasma beta is very similar to the well known case (the parameters were chosen in DZ in a way to reproduce solar wind conditions with increasing heliocentric distance, trying to keep the predicted $\gamma$ in a narrow range for ease of comparison).

In Figure 1 we plot the rms density fluctuations, the normalized cross helicity $\sigma=\left(E^{+}-E^{-}\right) /\left(E^{+}+E^{-}\right)$and the Elsässer energies $E^{ \pm}$(normalized to $E^{+}(0)$ ) for run A parameters and for $\theta=30^{\circ}$. Like in DZ multiple decays may be seen, at least in this low-beta case, where density fluctuations first increase and then saturate and where the dominant wave looses about half of its energy at every instability saturation. Correspondingly, the cross helicity reverses its sign flipping between the \pm 1 states for pure Alfvénic modes. The plots for run $\mathrm{B}$ and $\mathrm{C}$ are also very similar to those in DZ and are not reported here. The result that for $\beta \sim 1$ the asymptotic value of $\sigma$ approaches zero after the first occurrence of the instability still holds (B: $\sigma=-0.3$, C: $\sigma=0.2$ ).

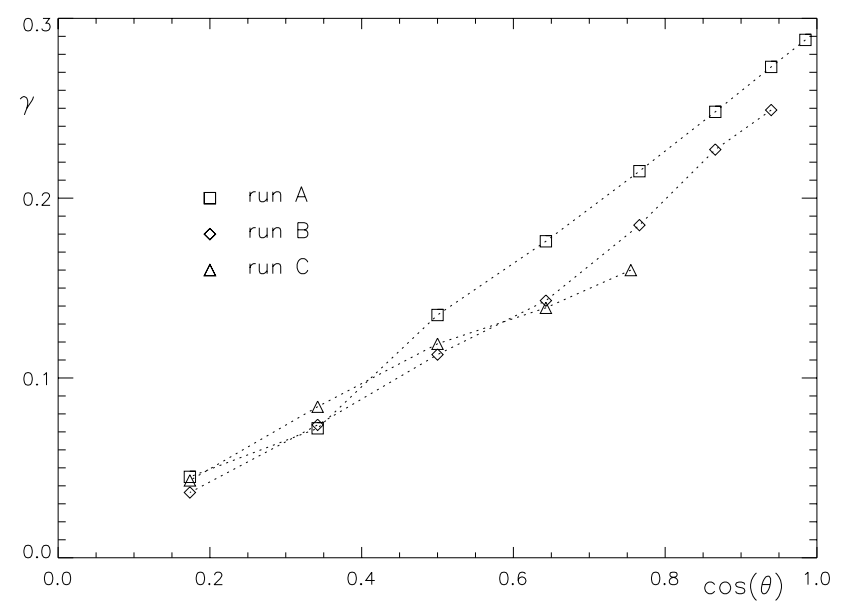

Figure 2. The measured growth rates $\gamma$ as a function of $\cos \theta$, with $\theta$ ranging from $10^{\circ}$ to $80^{\circ}$. 

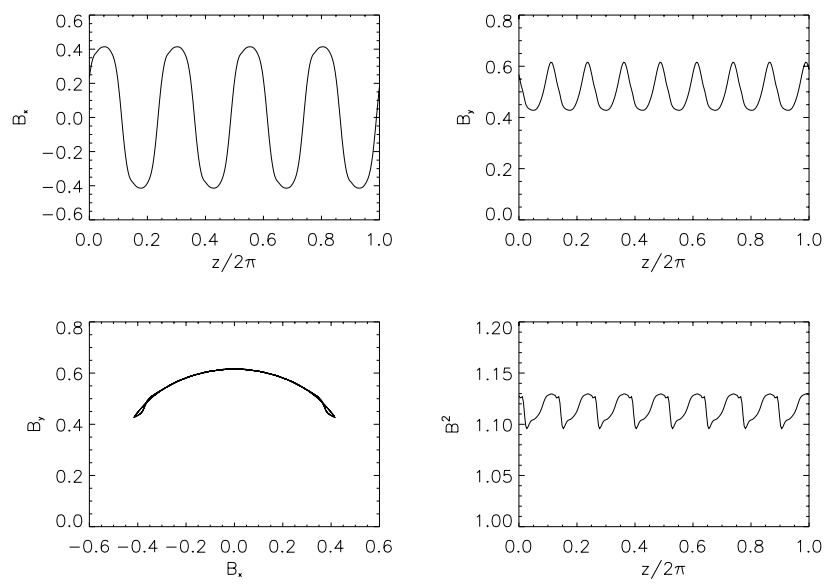

Figure 3. Magnetic field components $\left(B_{z}\right.$ is constant and it is not shown), hodogram in the $B_{x}-B_{y}$ plane and $B^{2}$ for $t=3 \pi$. Here $\eta=\beta=0.5$ and $\theta=30^{\circ}$.

In spite of the striking similarities between the behavior of circular and arc-polarized waves, the evolution of the instability is slower in the latter case. This is mainly due to the fact that the phase velocity is smaller by a factor $\cos \theta$, since the dispersion relation for the oblique mother wave is $\omega_{0}=k_{0} v_{A} \cos \theta$ and both times and growth rates should be normalized correspondingly. In Figure 2 the derived growth rates for a set of different angles are shown for the three runs (the critical angles in Eq. (3) are $\theta=7.31^{\circ}, \theta=18.56^{\circ}$, and $\theta=39.54^{\circ}$ for $\eta=0.2, \eta=0.5$, and $\eta=1.0$, respectively). The linear dependence on $\cos \theta$ is especially apparent for small amplitudes, as expected.

\subsection{Linearly polarized waves}

Consider now the evolution of an oblique Alfvénic mode linearly polarized along the $x$ direction, that is with fluctuations still defined by Eq. (1) but no components along $y$. This time $B^{2}$ is not constant and both a driven wave and magnetoacoustic waves, all with $k=2 k_{0}=8$, are expected to form (density noise at $t=0$ is not needed, due to initial non-equilibrium). The driven wave, that travels at the same speed as the mother wave, tends to restore a $B^{2} \simeq$ const condition by creating a $B_{y}$ component in arc polarization. The situation may be seen in Figure 3, where an almost exact arc with nearly constant total magnetic strength is achieved. The parameters are those of run B, $\theta=30^{\circ}$ and $t=3 \pi$. Note that the $B_{x}$ profile has almost phase-steepened to a RD, although this is much more apparent for larger amplitudes.

Density fluctuations first increase very rapidly due to the ponderomotive force, but soon steepen and dissipate through shock heating, causing the $r m s$ value to decrease. In general, for smaller $\beta$ and larger $\eta$ density fluctuations are larger, but also dissipate more rapidly. When this value reaches a noise level, the situation is very similar to that of the previous section because we have a traveling arcpolarized wave with an almost constant field strength: parametric decay finally sets in and the mother wave decays as usual (see Figure 4). The linear growth rates are about the same as those of the corresponding case of initial arc polarization, just around $10 \%$ less because of a stronger wave amplitude decay: for example $\gamma=0.120$ and $\gamma=0.093$ are found for the cases corresponding to runs A and B in Table 1. No decay is found for run C parameters, because the wave amplitude damping is too strong for $\eta=1$ and the instability interval does not contain integer wave numbers any longer.

Parametric decay was invoked by $\mathrm{VH}$ to explain the growth of the density fluctuations at $k=2 k_{0}$, in their lowbeta run, beyond the value reached by nonlinear steepening alone. This further growth was seen to saturate between one and two mother wave periods $P_{0}$, and this looks consistent with their estimate of a $\gamma / \omega_{0} \simeq 0.18$, that should lead to a characteristic growth time $\tau \simeq 0.88 P_{0}$ ( $\tau$ is so short because the amplitude here is $\eta=1$ ). Unfortunately, time histories of $r m s$ quantities like $\delta \rho$ or $\sigma$ were not provided and the typical signatures of parametric decay are harder to recognize. To further clarify this point we have run a simulation with the same parameters as in $\mathrm{VH}$, but we could not find a clear increase in $\delta \rho_{\text {rms }}$ beyond that due to the initial ponderomotive force (only at much larger times, as in Fig. 4), while nonlinear steepening and dissipation were clearly still at work. If the density fluctuations increase in $\mathrm{VH}$ is really due to parametric instability, the fact that we do not observe this effect might be due to dissipation of compressive steepened fronts, which is absent in hybrid codes where ion wave dispersion and ion kinetics should arrest the steepening. Another possibility might be that dissipation alter the instability range, but this is so broad for the chosen parameters (and the decrease in mother wave amplitude so small) that we tend to exclude this possibility.

\section{Summary and conclusions}

In this paper we have shown, through 1-D MHD simulations under a set of different physical conditions, that monochromatic large-amplitude Alfvén waves in oblique propagation with arc-type polarization (preserving a constant total field intensity) are subject to parametric decay, occurring qualitatively unchanged as in the case of parallel propagation and circular polarization (see DZ). The growth rates are measured for a set of parameters and for several different propagation angles. The observed values are smaller than in the circular polarization case, mainly because of the
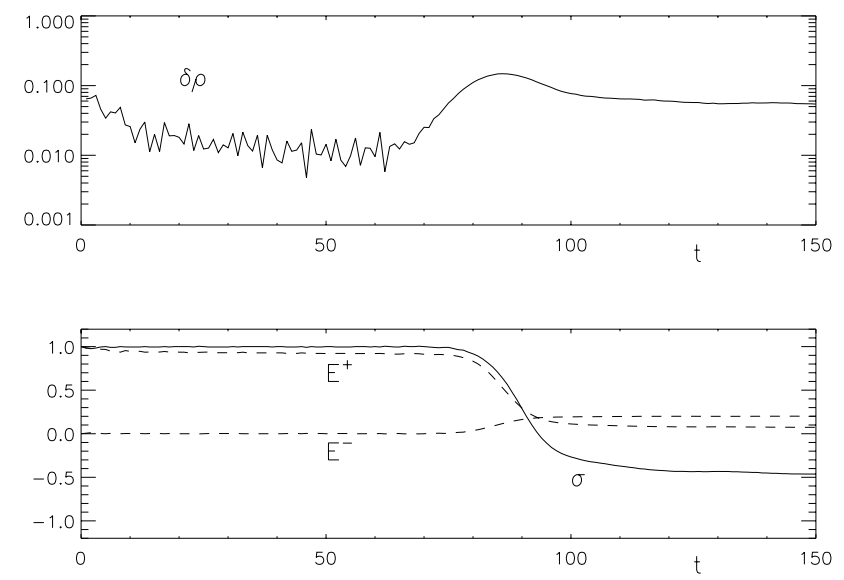

Figure 4. Same quantities as in Figure 1 but for the run of Figure 3, with a linearly polarized wave as initial condition. After an initial dissipation phase, parametric decay sets in and saturates around $t=85$. 
slower wave propagation ( $\gamma \sim \cos \theta$ for small amplitudes). Moreover, even the state of approximate arc polarization and constant magnetic field amplitude, which is reached after nonlinear evolution of initially linearly polarized oblique waves, appears to be unstable to parametric decay, that invariably occurs when the plasma beta is low or when the amplitude is large enough. Even multiple decays are sometimes found in the simulations, with cross helicity flipping between states of dominant pure inward or outward modes, usually in low-beta regimes, while for solar wind conditions the asymptotic state is that of an increasing balance between opposite propagating modes.

Parametric decay is certainly a slow process in solar wind conditions, and both partial reflection due to Alfvén speed gradients or interaction with velocity shear layers are able to shape the turbulent spectrum in much shorter timescales. However, if we consider that the fast solar wind (and particularly the polar wind) is extremely smooth and that the evolution of the Alfvénic ratio $r_{A}=E^{-} / E^{+}$and normalized cross helicity $\sigma$ occurs over distances $d \sim 1 \mathrm{AU}$ [Roberts et al., 1987; Bavassano et al., 2000], parametric decay may be then play the dominant role there. By taking our parameter sets $\mathrm{A}, \mathrm{B}$ and $\mathrm{C}$, that simulate different conditions from the outer corona to the Earth and for which the ratio $\gamma / \omega_{0} \sim 5 \%$ is constant (say it does not depend on the distance from the Sun), a wave with $P_{0} \sim 10^{4} \mathrm{~s}$ will decay with a characteristic $e$-folding distance $d \sim(1 / 2 \pi)\left(\gamma / \omega_{0}\right)^{-1} v_{\mathrm{SW}} P_{0} \sim 0.2$ AU for $v_{\mathrm{SW}} \sim 800 \mathrm{~km} / \mathrm{s}$, that has the correct order (it may be slightly larger when considering non-monochromatic waves or dispersive effects). Moreover, the Ulysses polar wind data by [Bavassano et al., 2000] show that the Alfvénic ratio ceases to increase at 2.5 $\mathrm{AU}$, its value being $r_{A} \sim 0.5 \Rightarrow \sigma=\left(1-r_{A}\right) /\left(1+r_{A}\right) \sim 0.3$, to be compared with our asymptotic value of $\sigma \sim 0.2$ for solar wind conditions. However, for more realistic modeling, non-monochromatic wave trains, dispersion and radial expansion should be included in the simulations.

Acknowledgments. The author thanks M. Velli, P. Londrillo and R. Grappin for several stimulating discussions.

\section{References}

Barnes, A., and J. V. Hollweg, Large-amplitude hydromagnetic waves, J. Geophys. Res., 79, 2302, 1974 (BH).
Bavassano, B., E. Pietropaolo, and R. Bruno, On the evolution of outward and inward Alfvénic fluctuations in the polar wind, J. Geophys. Res., 105, 15,959, 2000.

Del Zanna, L., M. Velli, and P. Londrillo, Parametric decay of circularly polarized Alfvén waves: Multidimensional simulations in periodic and open domains, Astron. Astrophys. 367, 705, 2001 (DZ).

Inhester, B., A drift-kinetic treatment of the parametric decay of large-amplitude Alfvén waves, J. Geophys. Res., 95, 10,525, 1990.

Leamon, R. J., W. H. Matthaeus, C. W. Smith, G. P. Zank, D. J. Mullan, and S. Oughton, MHD-driven kinetic dissipation in the solar wind and corona, Astrophys. J., 537, 1054, 2000.

Londrillo, P., and L. Del Zanna, High order upwind schemes for multidimensional magnetohydrodynamics, Astrophys. J., 530, $508,2000$.

Medvedev, M. V., V. I. Shevchenko, P. H. Diamond, and V. L. Galinsky, Fluid models for kinetic effects on coherent nonlinear Alfvén waves. II. Numerical solutions, Phys. Plasmas, 4, 1257, 2000.

Riley, P., C. P. Sonnet, A. Balogh, R. J. Forsyth, E. E. Scime, and W. C. Feldman, Alfvénic fluctuations in the solar wind: A case study using Ulysses measurements, Space Sci. Rev., 72, 197, 1995.

Riley, P., C. P. Sonnet, B. T. Tsurutani, A. Balogh, R. J. Forsyth, and G. W. Hoogeveen, Properties of arc-polarized Alfvén waves in the ecliptic plane: Ulysses observations, J. Geophys. Res., 101, 19,987, 1996.

Roberts, D. A., M. L. Goldstein, L. W. Klein, and W. H. Matthaeus, Origin and evolution of fluctuations in the solar wind: Helios observations and Helios-Voyager comparisons, $J$. Geophys. Res., 92, 12,023, 1987.

Tsurutani, B. T., and C. M. Ho, A review of discontinuities and Alfvén waves in interplanetary space: Ulysses results, Rev. Geophys., 37, 517, 1997.

Vasquez, B. J., and J. V. Hollweg, Formation of arc-shaped Alfvén waves and rotational discontinuities from oblique linearly polarized wave trains, J. Geophys. Res., 101, 13,527, 1996b (VH).

L. Del Zanna, Dipartimento di Astronomia e Scienza dello Spazio, Università di Firenze, Largo E. Fermi 5, I-50125 Firenze, Italy (ldz@arcetri.astro.it).

(Received January 24, 2001; revised March 27, 2001; accepted April 20, 2001.) 